\title{
A Comprehensive Study of Bone Manifestations in Adult Gaucher Disease Type 1 Patients in Argentina
}

\author{
Beatriz Oliveri $^{1}$ [ $\cdot$ Diana González $^{2} \cdot$ Felisa Quiroga $^{3} \cdot$ Claudio Silva $^{3} \cdot$ Paula Rozenfeld $^{4}$
}

Received: 25 July 2018 / Accepted: 12 February 2019 / Published online: 21 February 2019

(c) Springer Science+Business Media, LLC, part of Springer Nature 2019

\begin{abstract}
Gaucher disease (GD) is the most prevalent lysosomal storage disease, and bone involvement is the most disabling condition. The aim of the present study was to evaluate bone involvement in adult patients with GD, using an observational cross-sectional study. Patients were evaluated using X-rays, bone densitometry (BMD), trabecular bone score (TBS), magnetic resonance imaging (MRI), and biochemical bone markers. Thirty-two type 1GD patients were included (mean age: $40 \pm 16$ years). Patients had received velaglucerase for $2.7 \pm 1.4$ years; $19 / 32$ had been treated previously with imiglucerase. Ninety-four percent of subjects met therapeutic goals for hematological parameters, and eight were splenectomized (SPX). Nineteen patients had irreversible bone lesions (IL), i.e., avascular necrosis, bone infarction, and/or vertebral fractures. MRI showed marrow infiltration in $71 \%$ of patients. Patients with IL had higher bone marrow burden than those without $(p=0.001)$. All SPX patients had IL, a higher prevalence of bone marrow edema $(p=0.02)$, and lower TBS $(p=0.03)$ than non-SPX patients. Only $18.7 \%$ of patients had abnormal BMD, with no correlation with fractures (FX). TBS values were $<1350$ in $53 \%$ of patients and tended to be lower in those with FX $(p=0.06)$. Patients with P1NP in the lower quartile had lower TBS $(p=0.03)$ than those with P1NP in the higher quartiles. TBS correlated moderately but not significantly with P1NP $(r=0.32)$ and BMB $(r=-0.44)$. A high prevalence of IL was documented. Bone quality was more affected than BMD in fracture patients. Low bone formation, active bone marrow infiltration, and splenectomy might be implicated in IL.
\end{abstract}

Keywords Gaucher disease · Skeletal complications · Velaglucerase $\cdot$ Bone involvement · Bone quality or TBS · Bone markers

\section{Introduction}

Gaucher disease (GD) (GD, OMIM \#230,800, ORPHA355) is the most prevalent inherited lysosomal storage disease and is caused by mutations in GBA gene that confer a deficient

Beatriz Oliveri

beatrizoliveri258@gmail.com

1 Laboratorio de Osteoporosis y Enf. Metabólicas Oseas, INIGEM (UBA-CONICET) Hosp.de Clínicas JSM, Cordoba 2351-Piso 8, 1120 Ciudad Autonoma de Buenos Aires, Argentina

2 Mautalen Salud e Investigación, Azcuenaga, 1860-Piso 6, 1128 Ciudad Autonoma de Buenos Aires, Argentina

3 Diagnóstico Maipú, Av. Maipú 1660, Vicente López, 1602 Buenos Aires, Argentina

4 IIFP, Universidad Nacional de La Plata, CONICET, Facultad de Ciencias Exactas, Departamento de Ciencias Biologicas, Calle 47 y 115-La Plata, 1900 Buenos Aires, Argentina level of activity of glucocerebrosidase (GCase). This deficiency leads to accumulation of the glycolipid glucocerebroside in the lysosomes of cells of the monocyte/macrophage system [1,2]. Very rarely, GD may be caused by a mutation in the PSAP gene, leading to a deficiency in saposin $\mathrm{C}$ without GCase deficiency [3].

Visceromegalies, bone involvement, and peripheral cytopenias are described especially in type $1 \mathrm{GD}$, which has an estimated global prevalence of 1 in 40,000-60,000 people. In Ashkenazi Jewish descendants, however, prevalence may reach $0.05-0.1 \%$ [2].

GD has highly heterogeneous clinical presentation, due in part to the large number of GCase gene mutations.

According to the International Collaborative Gaucher Group Registry (ICGGR) [4], one-sixth of GD patients are estimated to live in Latin America. A significantly higher proportion of female patients has been observed in this region as compared to other areas. Similarly, mean age of diagnosis and proportion of patients with radiological 
findings are lower in Latin America as compared to other continents. However, bone marrow infiltration rates seem to be similar in this region and the rest of the world [4]. The proportion of any bone involvement has been estimated at 80\% in GD patients in Latin America, whereas this percentage seems slightly higher in other regions when considering all imaging techniques [4].

In the context of such clinical presentation heterogeneity, adequate characterization of GD is essential for a correct treatment approach. Evaluation of GD burden in patients receiving enzymatic replacement treatment (ERT) includes analysis of the potential relationship among demographic, clinical, and complementary test data, especially in terms of skeletal GD manifestations [5]. In view of the slower skeletal response to ERT as compared to hematological and visceral changes [6], the occurrence of irreversible lesions (IL) that have a high impact on quality of life [7], and discrepancies regarding the correlation between skeletal involvement and hematological/visceral involvement [8], there is a strong need for studies that thoroughly evaluate bone involvement.

Based on the above, the aims of the present study were to describe bone involvement in a group of adult patients with Type $1 \mathrm{GD}$, and to investigate the potential relationship between the severity of bone involvement and the different parameters of bone status and GD markers studied here. Of note, in addition to conventional bone parameters, trabecular bone score (TBS) was also used in the present study to assess bone quality.

\section{Patients and Methods}

A cross-sectional, descriptive analysis was conducted using a prospectively collected database of velaglucerase-treated GD patients in Argentina. Adult patients (age $\geq 20$ years) were entered on the database following referral from hematology specialists for evaluation of skeletal involvement, between December 2015 and February 2017.

All patients had GD confirmed by B GCase activity assay. All studies were performed at the same institution, with the same nuclear magnetic resonance, X-ray, and densitometry equipment.

The following data were collected from all subjects: age, gender, self-reported ethnicity, GBA1 gene analysis, signs and symptoms that prompted consultation, time from diagnosis of GD, ERT prior to velaglucerase treatment, and symptoms at the time of the study.

The presence of skeletal alterations was evaluated using plain radiographs of the dorsal and lumbar spine (LS) and of the limbs, and magnetic resonance imaging (MRI) of the spine, femurs, and total skeleton (Philips Achiva Pulsar SE 1.5T MRI Scanner). T1-weighted MRI detects and quantifies the extent of marrow infiltration, and T2-weighted MRI identifies focal lesions. Acute pathology such as bone marrow edema, acute bone infarcts (BI), and avascular necrosis $(\mathrm{AVN})$ are detected with $\mathrm{T} 2$ with fat saturation. The sclerotic bone stores seen in simple X-ray examinations were interpreted as BI if they also appeared as hypointense foci in MRI.

Vertebral fractures (FX) were diagnosed on lateral lumbar and dorsal radiographs according to the semiquantitative method described by Genant [9].

Bone mineral density (BMD) was measured at LS $\left(\mathrm{L}_{1}-\mathrm{L}_{4}\right)$ and femur $(\mathrm{F})$, using DXA imaging (LUNAR Prodigy Advance, version 14.10.022). Variation coefficients for LS and F BMD were $1.3 \%$ and $1.4 \%$, respectively.

BMD results were expressed in absolute values $\left(\mathrm{g} / \mathrm{cm}^{2}\right)$, and as $Z$-scores and $T$-scores; $Z$-score was calculated as standard deviation score from age and sex-matched population and $T$-score as standard deviation score from normal reference population database. According to the ISCD guidelines, $Z$-scores are used in patients under the age of 50 years [10], and $T$-scores are used in patients above the age of 50 [11]. $T$-scores were classified as normal/osteopenia/ osteoporosis according to the WHO Technical report, and $Z$-scores were classified as normal/low bone mass according to ISCD official positions.

Bone quality was assessed by measuring TBS on two dimensional LS DXA images obtained using the densitometer described above. TBS is a texture analysis that is automatically calculated from DXA-LS images using the TBS iNsight software Medimaps Group SA (coefficient of variation: 4.6\%) [12]. Normal and abnormal scores have been established: TBS $\leq 1.2$ defines degraded microarchitecture, TBS between 1.200 and 1.350 defines partially degraded microarchitecture, and TBS $\geq 1.350$ is considered normal [12].

Bone marrow burden (BMB) is a semiquantitative scoring system that seeks to assess bone marrow involvement taking into account bone marrow infiltration in the LS and femur. It incorporates not only the visual interpretation of signal intensity ( $T 1$ and $T 2$ relaxation time), but also the geographic location in femur (proximal/distal epiphysis and/ or diaphysis) and pattern of bone marrow infiltration of LS (patchy or diffuse, and absence of fat in the basivertebral vein region). This scoring system assigns up to eight points for femoral involvement and eight points for LS involvement. Total BMB is obtained by adding femur and spine scores with maximum composite BMB score of 16 points (range 0-16). Higher scores reflect greater marrow infiltration [13]. BMB score determinations were performed by the same observer, thus avoiding interobserver variability. A significant $0.89(p=0.01)$ intra-observer correlation was observed.

The collected biochemical data included complete blood count, proteinogram, ferritin (FT), angiotensin converting 
enzyme (ACE), total calcemia, intact parathormone (PTH), 25-hydroxy-vitamin $\mathrm{D}$, and 2-h fasting urinary calcium-tocreatinine ratio $(\mathrm{Ca} / \mathrm{Cru})$ according to standard laboratory methods. Chitotriosidase (CHIT) activity was determined using a fluorometric method (inter-assay and intra-assay coefficients of variation were $9 \%$ and 5\%, respectively) [14].

Bone turnover was evaluated using three biochemical markers: C-terminal telopeptide of type I collagen (beta cross laps [CTX]) measured by electrochemiluminescence Elecsys (Roche) to investigate bone resorption (intra- and inter-analysis variation coefficients: $2.1 \%$ and $2.4 \%$, respectively), $\mathrm{N}$ terminal propeptide of procollagen type 1 (P1NP) measured by electrochemiluminescence Elecsys (Roche) (intra- and inter-analysis variation coefficients: $1.8 \%$ and $4.3 \%$, respectively), and bone alkaline phosphatase (BAP) by enzyme immunoassay (Abbot) (intra- and inter-analysis variation coefficients: 4.8 and $7.2 \%$, respectively) to investigate bone deposition and mineralization.

Analysis of the relation between severe IL and the parameters studied here only included BI, AVN, and vertebral FX, since these parameters are associated with the most deleterious skeletal complications and most affect patients' quality of life; hence, EM was excluded from the analysis.

Statistical analysis included descriptive statistics of clinical and imaging data in order to characterize bone involvement in type $1 \mathrm{GD}$ patients. Quantitative variables were first analyzed by calculating mean, standard deviation, median, and interquartile range (IQR) when necessary. Qualitative variables were characterized by frequency distribution. In order to provide appropriate statistical power, some quantitative variables were dichotomized into categorical variables. A Chi-square test was used for testing relationships between categorical variables. Nonparametric contrast tests were used for analyzing quantitative variables. A two-tailed $p$ value $<0.05$ was considered significant. Statistical analysis was performed using Excel® 2013 (Microsoft Corporation).

\section{Results}

A total 32 patients (19 female and 13 male patients) were studied. Mean age (mean \pm SD) was $40 \pm 16$ years (range 20-77).

GBA1 gene analysis was performed in 31 patients: RecNcil/C.1226A $>\mathrm{G}$ compound heterozygosity was found in $8 / 31(25.8 \%), C .1226 \mathrm{~A}>\mathrm{G} / 1448>\mathrm{G}$ compound heterozygosity in 7/31 (22.6\%), and C.1226A $>$ G/C.1226A $>$ G homozygosity in 6/31 (19.3\%), whereas the remaining $29 \%$ of patients had a combination of RecNcil, C.1226A > G, or $1448>\mathrm{G}$ with other alleles.

Only three patients were Ashkenazi Jewish descent, and the rest were of Caucasian or South American descent in similar percentages.
The median age at diagnosis was 22 years (IQR: 18.5 years), and age range was $1-47$ years of age. Thirteen patients (48\%) were diagnosed before the age of 20 years. Mean time from diagnosis was 17.5 years (range 1.5-45).

Main chief complaints that prompted consultation in $65 \%$ of patients included visceromegalies, cytopenia, and cytopenia-related manifestations; of note, $25 \%$ of patients reported bone pain and/or bone crisis at the time of consultation. Bone crisis is a sudden onset, self-limiting, exquisitely painful event, associated with signs of acute local and/or systemic inflammation. Bone crisis is typically but not necessarily associated with local tenderness, erythema, and swelling, and it often occurs with fever, and accelerated erythrocyte sedimentation rate.

At enrollment in the study, all patients had been receiving velaglucerase for $2.7 \pm 1.4$ years, and 19 out of 32 patients had been treated previously with imiglucerase.

A total of 29/32 (90\%) and 30/32 (94\%) had achieved treatment goals (TG) for thrombocytopenia and anemia, respectively, according to current Argentinian recommendations [15]. Liver volumetry data were obtained from 30 patients because two patients had refused to have an MRI, and spleen volumetry data were obtained from 22 patients since eight had previously undergone splenectomy. Only one patient failed to reach TG for visceromegalies, presenting moderate hepatomegaly ( $>1.5$ times the normal liver volume) and severe splenomegaly ( $>15$ times the normal spleen volume).

According to the medical history data collected in the present study, nine patients reported a history of long bone fractures, none reported having suffered a bone crisis within the year prior to enrolment in the study, and nine patients reported osteoarticular pain at the time of the study, which was interpreted as secondary to AVN, joint replacement, or vertebral FX.

\section{Radiological Alterations}

Radiological alterations were observed in 27/32 patients (84.4\%) (Table 1). Erlenmeyer flask deformity was the most prevalent alteration ( $70 \%$ of subjects with positive radiological findings). In seven patients with no history of clinical vertebral FX, diagnosis of morphometric vertebral FX was established according to the semiquantitative visual assessment [9].

\section{MRI Findings}

Bone marrow infiltration was the most prevalent anomaly found in MRI, followed by IL, BI, and AVN (Table 2).

Results of BMB score calculated in the 23 patients with bone marrow infiltration in femurs and in the seven patients with infiltration in the LS, showed a median LS BMB score 
Table 1 Main radiological findings $(n=27)$

\begin{tabular}{lc}
\hline Erlenmeyer flask deformity & $19 / 27(70 \%)$ \\
Lytic lesions & $15 / 27(55.5 \%)$ \\
Avascular necrosis & $11 / 27(40.7 \%)$ \\
Heterogeneous bone architecture & $15 / 27(55.5 \%)$ \\
Sclerotic lesions & $11 / 27(40.7 \%)$ \\
Morphometric vertebral fractures & $7 / 27(26 \%)$ \\
Prosthesis & $6 / 27(22.2 \%)^{*}$ \\
Bone infarction $* *$ & $13 / 27(48 \%)$ \\
\hline
\end{tabular}

Percentages do not add $100 \%$ due to overlap of radiological manifestations in several subjects

*Four patients had undergone hip replacement and two patients had both a hip and knee prosthesis; **sclerotic lesions suggestive of BI and confirmed by MRI

Table 2 MRI findings

\begin{tabular}{lll}
\hline Parameters & $n$ & $\%$ \\
\hline $\begin{array}{l}\text { Positive findings } \\
\text { Irreversible lesions }\end{array}$ & $23 / 30^{\mathrm{a}}$ & 76.7 \\
$\quad$ Avascular necrosis & $14 / 23$ & 60.8 \\
$\quad$ Bone infarction & $16 / 23$ & 69.6 \\
Reversible lesions & & \\
$\quad$ Bone marrow infiltration, femurs & $23 / 23$ & 100 \\
Bone marrow infiltration, lower limbs (except & $16 / 23$ & 66.6 \\
$\quad$ femurs) & & \\
Bone marrow infiltration, upper limbs & $14 / 23$ & 60.8 \\
Bone marrow infiltration, spine & $7 / 23$ & 30.4 \\
Bone marrow infiltration, sacrum and/or iliac bone & $3 / 23$ & 13.0 \\
Bone edema & $9 / 23$ & 39.1 \\
\hline
\end{tabular}

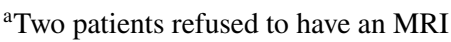

of 6; IQR 3.5 (range 3-8). All patients with spine infiltration also had positive BMB in the femurs. Median femoral BMB score was 5.5; IQR 2 (range 2-8). Median total BMB (LS + femurs) was five, IQR 4.75 (range 2-15). Residual irreversible complications (BI, AVN, and FX) were observed in 19 patients, all of whom had femoral marrow infiltration. Thus, marrow infiltration in the femur was significantly more frequent in IL patients as compared to only five patients with femoral marrow infiltration in the group of patients without IL $(p=0.001)$. Femoral BMB score was higher in the group with IL compared to the group without IL $(p<0.001)$. No differences in densitometric or biochemical results were observed between patients with and without IL.

IL were observed in $100 \%$ of SPX patients, compared to a significantly lower $45 \%$ in non-SPX patients $(p=0.02)$, and a significantly higher prevalence of bone marrow edema was observed in SPX patients (5/8) as compared to nonSPX patients $(4 / 22)(p=0.02)$. Bone marrow infiltration in the femur affected all SPX patients and $69 \%$ of non-SPX patients. Femoral BMB of SPX patients was significantly higher than that of non-SPX patients $(6 \pm 0.95$ vs. $4 \pm 2.5$, respectively, $p<0.01)$. Bone marrow infiltration in the LS was observed in $57 \%$ of SPX patients, but in only $13 \%$ of non-SPX patients.

\section{Densitometric Results}

Although DXA scan was performed in all patients, femoral analysis of seven patients was excluded due to the presence of AVN or hip replacement $(n=5)$, or artifactual increase probably due to sclerotic lesions $(n=2)$. The abnormally increased spine value of one patient was also excluded. Considering the entire study population, only six out of 32 patients had abnormal BMD measurements for gender and age $(18.7 \%)$. None of the ten male patients younger than 50 years or of the 14 premenopausal female patients had $Z$-scores lower than -2 , which is considered the limit for low bone mass for that age group (10). Among the eight patients older than 50 years, five had osteopenia and one had osteoporosis ( $Z$-score and $T$-score values are shown in Table 3). Mean TBS was $1344 \pm 118$ (mean \pm SD), with values lower than 1350 in $53 \%$ of patients. No differences in TBS values were observed between male and female patients. There was no significant difference in TBS when comparing patients with and without IL and/or AVN. However, TBS values tended to be lower in the 12 fracture patients compared to the 20 patients without $\mathrm{FX}(p=0.06)$.

Table 3 Densitometric results

\begin{tabular}{lllll}
\hline BMD & $n$ & Median IQR & Range & \\
\hline Lumbar spine $Z$-score & 23 (aged $<50$ years) & -0.3 IQR 1.65 & $-1.5-+2.0$ & \\
Lumbar spine $T$-score & 8 (aged $>50$ years) & -1.4 IQR 0.55 & $-2.2--0.7$ & Osteopenia: $5^{*}$ \\
Femur $Z$-score & 20 (aged $<50$ years) & -0.2 IQR 1.15 & $-1.8-+1.6$ & \\
Femur $T$-score & 5 (aged $>50$ years) & -0.7 IQR 1.52 & $-3.1--0.1$ & Osteopenia: $1^{*}$ \\
& & & & Osteoporosis: \\
& & & & $1^{* *}$ \\
\hline
\end{tabular}

*Osteopenia $=$ when $T$-score is between $<-1.0$ and $>-2.5$; **osteoporosis $=$ when $T$-score is $\leq-2.5$ $I Q R$ interquartile range 
No correlation was observed between TBS and LS and F BMD. Nevertheless, patients with a spine $Z$-score $<-1$ had significantly lower TBS values than those with a $Z$-score $>-1(1273+/-90.7$ vs. $1387+/-115,2$; $p<0.03$ ).

TBS was lower in SPX than in non-SPX patients $(1299+112$ vs. $1378+111)$, whereas LS BMD $\left(\mathrm{g} / \mathrm{cm}^{2}\right)$ was similar $(1097+0.15$ vs. $1096+0.16)$.

\section{Biochemical Results}

Levels of 25-hydroxy-vitamin D were desirable ( $\geq 30 \mathrm{ng}$ / $\mathrm{ml}$ ) in 13 patients, insufficient (20 to $29.9 \mathrm{ng} / \mathrm{ml}$ ) in 13 , and deficient $(<20 \mathrm{ng} / \mathrm{ml})$ in six patients. Intact PTH levels were higher than normal in three patients, all of whom had normal calcemia but vitamin D insufficiency $(n=1)$ or deficiency $(n=2)$.

Markers of GD and bone remodeling are summarized in Table 4.

Results showed TBS values below 1350 in all the patients with P1NP levels in the lower quartile, but in only $37 \%$ of patients with P1NP levels in the upper quartile $(p<0.03)$.

Patients with P1NP in the upper quartile had a higher TBS than those with P1NP in the lower quartile (1262 \pm 71 vs. $1404 \pm 161, p=0.031)$, and only $37 \%$ of patients had a TBS below 1350. In addition, $100 \%$ of patients with P1NP in the lower quartile had a TBS below $1350(p<0.03)$. Patients with bone formation markers (P1NP and/or BAP) in the lower quartile showed greater bone marrow involvement (evidence of infiltration or edema on the MRI), though the increase did not reach statistical significance.

Table 4 Biomarkers of GD and bone remodeling markers

\begin{tabular}{|c|c|c|c|c|}
\hline Parameter & $n$ & Reference values & Mean & Range \\
\hline $\begin{array}{l}\text { Chitotri- } \\
\text { osidase } \\
\text { activity }\end{array}$ & $28 * \mathrm{a}, \mathrm{c}$ & $\begin{array}{l}7.77-110.50 \mathrm{mmol} / \\
\mathrm{ml} / \mathrm{h}\end{array}$ & $263.3 * *$ & $65.5-3039$ \\
\hline Ferritin & $31^{\mathrm{c}}$ & $\begin{array}{l}\text { Male: } 4.6-204 \mathrm{ng} / \mathrm{ml} \\
\text { Female: } 21.8- \\
274.7 \mathrm{ng} / \mathrm{ml}\end{array}$ & $\begin{array}{l}82 \pm 305 \\
355.9 \pm 558\end{array}$ & $\begin{array}{l}19.1-933 \\
43.1-1997\end{array}$ \\
\hline ACE & $29^{* \mathrm{~b}}$ & $8-52 \mathrm{U} / 1$ & $46.9 \pm 61.72$ & $8-250$ \\
\hline CTX & 32 & $0.30-0.58 \mathrm{ng} / \mathrm{ml}$ & $0.47 \pm 0.23$ & $0.13-1.01$ \\
\hline P1NP & $31^{*}$ & $20-85 \mathrm{ng} / 1$ & $65.5 \pm 22.58$ & $37.2-118$ \\
\hline FAO & $31^{*}$ & 31-95 IU/1 & $47.1 \pm 27.84$ & $11.4-110$ \\
\hline
\end{tabular}

*An outlier patient (Grubbs test) was detected and excluded from statistical analysis; $* *$ median

${ }^{\text {a }}$ Two patients were excluded due to homozygosity for the 24-bpduplication mutation in CHIT1 gene

${ }^{\mathrm{b}}$ Two patients under treatment with ACE inhibitor were excluded

${ }^{\mathrm{c}} 1$ missing data

\section{Discussion}

The present study sought to carry out a comprehensive evaluation of skeletal involvement in adult GD1 patients, including assessment of bone mass and quality and of bone markers, and analysis of skeletal lesions using radiological and MRI methods. Although most of the patients studied here had achieved the therapeutic goals for splenomegaly and hepatomegaly [15], we found a high prevalence of bone lesions (84\%), $70 \%$ of which were irreversible (EM, BI, AVN, and vertebral FX). Previously reported studies in series of untreated patients and treated adults as well as pediatric studies have shown a similar prevalence of bone lesions, ranging from 80 to $94 \%$ [4, 5, 16-19].

Pathological FX are usually associated with BI, and AVN leads to joint collapse, chronic pain, and disability [20]. Six patients in the present study had undergone hip or knee replacement, including two patients with both knee and hip arthroplasty.

Morphometric vertebral FXs were detected in 26\% of patients with no known history of vertebral FX. The mean age of patients with vertebral FX was 31 years (range 20-73), and 57\% were $<31$ years of age. Kahn et al. found that the most frequent FX localization in GD1 was the spine and that all fractures in GD1 occurred at a young mean age (29-57 years of age) [21].

Epidemiological studies suggest that a prevalent vertebral deformity increases the risk of subsequent vertebral deformities 7-10-fold and increases the risk of any FX 2.8 -fold $[22,23]$. Longitudinal studies are necessary to evaluate whether GD1 patients with fractures are also at an increased risk of future fractures.

Our patients reported no bone crisis within the year prior to treatment. This is an important therapeutic goal for skeletal disease, although $25 \%$ of patients reported osteoarticular pain secondary to AVN or vertebral FX, other unwanted consequences of IL.

We found no association between IL and severity of visceral or hematological diseases or GD biomarkers. SPX patients were the most affected by severe bone complications and showed higher femoral and lumbar BMB and a higher frequency of bone edema than non-SPX patients. This observation is in line with a well-known association between splenectomy and skeletal lesions [5, 24]. All the patients with severe IL had femoral marrow infiltration and higher femoral BMB scores than patients without IL. The high bone marrow infiltration documented by MRI is in line with the proposed mechanism that the progressive accumulation of glucocerebrosides within the bone marrow cavity induces a centrifugal expansion of red bone marrow, resulting in vascularity alterations, elevated intraosseous pressure, thrombosis, infarction, and AVN. 
Besides these mechanical consequences, macrophage activation enhances the production of inflammatory factors and cytokines that affect normal bone remodeling expressing the interaction among bone marrow, bone, and immune cells implicated in this complex mechanism [6, 14, 25].

No differences in densitometric parameters were observed between patients with and without IL. Only six out of 32 patients had abnormal BMD measurements for gender and age, according to the ISCD guidelines (two men older than 50 years and four postmenopausal women). Decreased bone mass is a prognostic risk factor for bone fractures in the general population [26]. Nevertheless osteoporotic fractures have also been reported in patients with normal BMD or with mild osteopenia, indicating that other factors such as bone microarchitecture and strength must be taken into account. TBS is a novel tool that provides information about trabecular microarchitecture. It has been reported that low TBS values may be a potential FX predictor independent of both clinical risk factors and LS and F BMD values [27, 28]. We found TBS values below 1.350 in $53 \%$ of patients, showing greater degradation of bone quality than of bone mass. TBS values tended to be lower in fracture patients than in those without fractures. SPX patients, who were the most affected by severe bone lesions, had lower TBS than non-SPX patients, whereas both groups had similar LS BMD values. We found no correlation between TBS and LS and F BMD, indicating that these two studies evaluate different bone properties. Based on reports by Khan et al. showing that GD patients with LS BMD Z-scores lower than -1 had higher risk of fractures [21], patients with LS BMD $Z$-score $<-1$ were analyzed separately and were found to have significantly lower TBS values than patients with BMD $Z$-score $\geq-1$.

There is increasing evidence that, irrespective of densitometric findings, TBS might provide useful information about bone health in conditions of secondary osteoporosis [29].

In the last 2 years, altered trabecular microarchitecture and hip geometry measured by TBS and hip structural analysis [30], and altered bone strength measured by impact microindentation have been reported in GD patients [31].

In line with reports published in the literature, 25-hydroxy-vitamin D deficiency and insufficiency were common features in our cohort of patients $[6,32]$. However, only three of those patients presented secondary hyperparathyroidism.

In a normal state, bone formation and resorption are usually closely coupled and generally balanced, allowing bone renewal and preservation of bone strength. Several studies suggest that bone turnover biomarkers may be disturbed in GD [33, 34]. Markers of bone formation are usually normal or decreased, whereas markers of bone degradation are normal or increased [6, 33,34]. In the present study, markers of bone remodeling did not differ significantly between patients with and without skeletal involvement. However, analysis of the distribution of bone remodeling markers showed that TBS values of patients with bone formation marker P1NP levels in the lower quartile were lower than those of patients with P1NP levels in the upper quartile and below 1350, indicating partially degraded microarchitecture [12]. In addition, prevalence of bone edema and bone marrow infiltration tended to be higher in patients with both BAP and P1NP in the lower quartile. This could suggest an influence of bone marrow infiltration by Gaucher cells on bone remodeling, more specifically on bone formation.

Based on the association between bone formation markers in the lower quartile, low TBS, and prevalence of bone edema and bone marrow infiltration in the GD patients observed here, it could be hypothesized that high bone marrow infiltration may lead to inhibition of bone formation and consequently to alterations in bone microarchitecture, resulting in poor bone quality. It was shown that induced pluripotent stem cell derived osteoblasts from GD patients exhibited reduced expression of osteoblast differentiation markers and defective mineral deposition [35]. Osteopenia caused by reduced bone formation was demonstrated in a murine model of GD [36]. However, other in vitro models have shown enhancement of bone resorption [37, 38].

One of the limitations to any study on rare diseases is the lack of an adequate number of patients due to the low incidence of the disease. We are aware that one of the major drawbacks of our analysis is the small number of enrolled patients, even when considering that Gaucher is a rare disease. Another limitation to the present study is its crosssectional design and lack of baseline studies at the time of diagnosis. Thus, it cannot be ascertained whether the bone lesions occurred before or during treatment with enzyme replacement.

The strength of our study is that all determinations were performed using the same equipment, and X-rays and MRI studies were analyzed by a single experienced observer [39]. In addition, the bone markers analyzed here are the markers proposed by an international consensus for evaluation of bone remodeling [40].

\section{Conclusion}

Even after reaching hematological TG, a high prevalence of irreversible bone lesions was documented in this population of GD1 patients. Bone quality evaluated by TBS was more affected than BMD in fracture patients. Low bone formation, active bone marrow infiltration, and splenectomy might be implicated in the occurrence of IL. Larger prospective studies are warranted to confirm the potential role of TBS in the early detection of bone involvement and in monitoring ERT efficacy. 
Acknowledgements The authors thank the following haematologists who collaborated in the recruitment of the patients: Arizo A, Aznar M, Bolesina M, Fernandez Sasso D., Fernandez V, Fondevila C, Guelbert N, Meschengeiser S, Murieda B, Navarro G, Onelda G, Rocaspana A, Romero Maciel A, Ruiz A., Santini F, Sanchez G, Stivel M, Watman N.

Author Contributions OB participated in the study design, acquisition and analysis of data, and drafting and revision of the manuscript. GD participated in the acquisition and analysis of data and drafting and revision of the manuscript. SC and QF participated in acquisition of data, RP participated in acquisition of data and critical review of the manuscript.

\section{Compliance with Ethical Standards}

Conflict of interest Beatriz Oliveri, Diana González, and Paula Rozenfeld have received speaking honoraria from Shire; Felisa Quiroga and Claudio Silva declare no conflict of interest.

Human and Animal Rights and Informed Consent This study was approved by the Ethical Committee of IBYME (Instituto de Biología y Medicina Experimental, Argentina). All patients or their guardians provided written informed consent to participate in this study.

\section{References}

1. Stirnemann J, Belmatoug N, Camou F, Serratrice C, Froissart R, Caillaud C et al (2017) A review of Gaucher disease pathophysiology, clinical presentation and treatments. Int J Mol Sci 18:441

2. Futerman AH, Sussman JL, Horowitz M, Silman I, Zimran A (2004) New directions in the treatment of Gaucher disease. Trends Pharmacol Sci 25:147-151

3. Horowitz M, Zimran A (1994) Mutations causing Gaucher disease. Hum Mutat 3:1-11

4. Drelichman G, Linares A, Villalobos J, Cabello JF, Kerstenetzky M, Kohan RM, Martins AM. (2012) Gaucher disease in Latin America. A report from the Gaucher Disease International Registry and the Latin American Group for Gaucher Disease. Medicina 72:273-82

5. Deegan PB, Pavlova E, Tindall J et al (2011) Osseous manifestations of adult Gaucher disease in the era of enzyme replacement therapy. Medicine (Baltimore) 90:52-60

6. Marcucci G, Zimran A, Bembi B et al (2014) Gaucher disease and bone manifestations. Calcif Tissue Int 95:477-494

7. Giraldo P, Solano V, Pérez-Calvo JI, Giralt M, Rubio-Félix D, Spanish Group on Gaucher disease (2005) Quality of life related to type 1 Gaucher disease: Spanish experience. Qual Life Res 14(2):453-462

8. Wenstrup RJ, Roca-Espiau M, Weinreb NJ, Bembi M (2002) Skeletal aspects of Gaucher disease: a review. Br J Radiol 75(1):A2-A12

9. Genant HK, Wu CY, van Kuijk C et al (1993) Vertebral fracture assessment using a semiquantitative technique. J Bone Miner Res $8: 1137-1148$

10. https://www.iscd.org/official-positions/2nd-iscd-pediatric-posit ion-development-conference/

11. https://www.iscd.org/official-positions/6th-iscd-position-devel opment-conference-adult

12. Silva BC, Leslie D, Resch $\mathrm{H}$ et al (2014) Trabecular bone score: a noninvasive analytical method based upon the DXA image. J Bone Miner Res 29:518-530
13. Maas M, van Kuijk C, Stoker J et al (2003) Quantification of bone involvement in Gaucher disease: MR imaging bone marrow burden score as an alternative to dixon quantitative chemical shift MR imaging-initial experience. Radiology 229(2):554-561

14. Bondar C, Mucci J, Crivaro A, Ormazabal M, Ceci R, Oliveri B, González D, Rozenfeld P (2017) In vitro osteoclastogenesis from Gaucher patients' cells correlates with bone mineral density but not with chitotriosidase. Bone 103:262-269

15. Drelichman G, Fernández Escobar N, Basack N et al (2015) Argentina Gaucher disease consensus update: Argentine group for diagnosis and treatment of Gaucher disease. Hematologia 19:4-51

16. van Dussen L, Biegstraaten M, Dijkgraaf MGW, Hollak CEM (2014) Modelling Gaucher disease progression: long term enzyme replacement therapy reduces the incidence of splenectomy and bone complications. Orphanet J Rare Dis 9:112

17. Andrade-Campos M, Valero E, Roca M, Giraldo P (2018) On behalf of the, Spanish group on Gaucher disease: the utility of magnetic resonance imaging for bone involvement in Gaucher disease. Assessing more than bone crises blood cells, Mol Dis 68: 126-134

18. Kaplan P, Andersson HC, Kacena K, Yee JD (2006) The clinical and demographic characteristics of nonneuronopathic Gaucher disease in 887 children at diagnosis. Arch Pediatr Adolesc Med 160:603-608

19. Drelichman G, Fernández Escobar N, Basack N et al (2016) Skeletal involvement in Gaucher disease: an observational multicenter study of prognostic factors in the Argentine Gaucher disease patients. Am J Hematol 91:E448-E453

20. Mikosch P, Hughes D (2010) An overview on bone manifestations in Gaucher disease. Wien Med Wochenschr 160:609-624

21. Khan A, Hangartner T, Weinreb NJ et al (2012) Risk factors for fractures and avascular osteonecrosis in type 1 Gaucher disease: a study from the International Collaborative Gaucher Group (ICGG) Gaucher registry. J Bone Miner Res 27:1839-1848

22. Melton LJ, Atkinson EJ, Cooper C, O’Fallon WM, Riggs BL (1999) Vertebral fractures predict subsequent fractures. Osteoporos Int 10:214-221

23. Harvey N, Dennison E, Cooper C (2013) The epidemiology of osteoporotic fractures. In: Rosen C (ed) Primer on the metabolic bone diseases and disorders of mineral metabolism, 8th edn. Wiley-Blackell, New Jersey, pp 348-356

24. Crary S, Buchanan G (2009) Vascular complications after splenectomy for hematologic disorders. Blood 114:2861-2868

25. Mucci JM, Rozenfeld P (2015) Pathogenesis of bone alterations in Gaucher disease: the role of immune system. J Immunol. https ://doi.org/10.1155/2015/192761

26. Kanis JA (2002) Diagnosis of osteoporosis and assessment of fracture risk. Lancet 359(9321):1929-1936

27. Hans D, Goertzen AL, Krieg MA, Leslie WD (2011) Bone microarchitecture assessed by TBS predicts osteoporotic fractures independent of bone density: the Manitoba study. J Bone Miner Res 26:2762-2769

28. Harvey NC, Glüer CC, Binkley N, McCloskey EV, Brandi ML, Cooper $\mathrm{C}$ et al (2015) Trabecular bone score (TBS) as a new complementary approach for osteoporosis evaluation in clinical practice: a consensus report of a European Society for Clinical and Economic Aspects of Osteoporosis and Osteoarthritis (ESCEO) working group. Bone 78:216-224. https://doi.org/10.1016/j. bone.2015.05.016

29. Ulivieri FM, Silva BC, Sardanelli F, Hans D, Bilezikian JP, Caudarella R (2014) Utility of the trabecular bone score (TBS) in secondary osteoporosis. Endocrine 7:435-448

30. Baldini M, Casirati G, Ulivieri FM, Cassinerio E, Chalouhi KK, Poggiali E et al (2018) Skeletal involvement in type 1 Gaucher disease: not just bone mineral density. Blood Cells Mol Dis 68:148-152. https://doi.org/10.1016/j.bcmd.2017.06.003 
31. Herrera S, Perez-Lopez J, Molto-Abad M, Guerri-Fernandez G, Cabezudo E, Novelli S et al (2017) Assessment of bone health in patients with type 1 Gaucher disease using impact microindentation. J Bone Miner Res 32:1575-1581

32. Parisi MS, Mastaglia SR, Bagur A, Goldstein G, Zeni SN, Oliveri B (2008) Body composition and bone metabolism in young Gaucher disease type I patients treated with imiglucerase. Eur J Med Res 13:31-38

33. van Dussen L, Lips P, Everts VE et al (2011) Markers of bone turnover in Gaucher disease: modeling the evolution of bone disease. J Clin Endocrinol Metab 96:2194-2205

34. Giuffrida G, Cingari M, Parrinello N et al (2012) Bone turnover markers in patients with type 1 Gaucher disease. Hematol Rep 4:e21. https://doi.org/10.4081/hr.2012.e21

35. Panicker LM, Srikanth MP, Castro-Gomes T, Miller D, Andrews NW. Feldman RA (2018) Gaucher disease iPSC-derived osteoblasts have developmental and lysosomal defects that impair bone matrix deposition. Hum Mol Genet 27:811-822. https://doi. org/10.1093/hmg/ddx442

36. Mistry PK, Liu J, Yang M, Nottoli T, McGrath J, Jain D et al (2010) Glucocerebrosidase gene-deficient mouse recapitulates Gaucher disease displaying cellular and molecular dysregulation beyond the macrophage. Proc Natl Acad Sci USA 107:19473-19478
37. Reed M, Baker RJ, Mehta AB, Hughes D (2013) Enhanced differentiation of osteoclasts from mononuclear precursors in patients with Gaucher disease. Blood Cells Mol Dis 51:185-194

38. Mucci M, Scian R, De Francesco PN et al (2012) Induction of osteoclastogenesis in an in vitro model of Gaucher disease is mediated by $\mathrm{T}$ cells via TNF- $\alpha$. Gene 509:51-59. https://doi. org/10.1016/j.gene.2012.07.071

39. Lai JKC, Robertson PL, Goh C, Szer J (2018) Intraobserver and interobserver variability of the bone marrow burden (BMB) score for the assessment of disease severity in Gaucher disease, possible impact of reporting experience. Blood Cell Mol Dis 68:121-125

40. Vasikaran S, Eastell R, Bruyere O, Foldes AJ, Garnero P, Griesmacher A et al (2011) Markers of bone turnover for the prediction of fracture risk and monitoring of osteoporosis treatment: a need for international reference standards. Osteoporos Int 22:391-420. https://doi.org/10.1007/s00198-010-1501-1

Publisher's Note Springer Nature remains neutral with regard to jurisdictional claims in published maps and institutional affiliations. 